\title{
Pentalogy of Cantrell in Full Term Neonate
}

\author{
Nadereh Taee ${ }^{1}$ Mojgan Faraji Goodarzi ${ }^{1} \quad$ Mohammad Safdari $^{2}$ Amir Bajelan ${ }^{2}$ \\ ${ }^{1}$ Department of Pediatrics, Lorestan University of Medical Sciences, \\ Khorramabad, Iran \\ 2 Medical Student, Lorestan University of Medical Sciences, \\ Khorramabad, Iran \\ Address for correspondence Mojgan Faraji Goodarzi, MD, \\ Department of Pediatrics, Faculty of Medicine, Lorestan University of \\ Medical Sciences, Khorramabad 066, Iran \\ (e-mail: dr.m.f.goodarzi@gmail.com).
}

Am J Perinatol Rep 2019;9:e144-e146.

\begin{abstract}
Keywords

- Pentalogy of Cantrell

- omphalocele

- pulsatile mass

Pentalogy of Cantrell (PC) is an uncommon congenital disorder characterized by severe defects in the chest and abdomen, including abdominal visceral prolapsed via umbilical cord (omphalocele), defect in the lower part of the sternum, defect in the front of the diaphragm, defects in the anterior part of the pericardium, and the ectopiacordis. Here, we report a 2-hour-old girl, weighing 3,500 g, who was referred to Shahid Madani Hospital in Khorramabad due to the large omphalocele on her chest with pulsating mass above it. The baby was the first child of a 24-year-old mother who was born with an uncomplicated vaginal delivery. Very rare cases of PC are born as a term new-born.
\end{abstract}

Pentalogy of Cantrell (PC) is a congenital disorder characterized by hallmark of omphalocele and ectopia cordis. PC includes extreme defects of the chest and abdomen including omphalocele, defect of the lower part of the sternum, defect of the front of the diaphragm, defect of the anterior portion of the pericardium, and cardiac abnormalities.

This syndrome occurs in different variation from incomplete to complete form with involvement in other organs (limbs, head and neck, and kidneys). ${ }^{1}$ The complete form of $\mathrm{PC}$ is very unusual. The pathogenesis of syndrome is unknown. Evolutionary defects of the mesoderm layer of embryogenesis onset on 14 to 18 days after conception are known to be effective in this syndrome.

This abnormality was first described in $1958 .^{2}$ It is part of the main upper coelosomies. The PC has five cardinal malformations including the supra umbilical hernia of the abdominal wall, a defect of the diaphragmatic pericardium, a defect of the anterior part of the diaphragm, an abnormality of the lower part of the sternum, and the malformation of the heart. ${ }^{3}$ There is a possibility of antenatal diagnosis from the 12th gestational week using ultrasound. The medical termination of pregnancy (MTP) remains the therapeutic option in some cases since prognosis is poor. In this study, we report a 2-hour-old girl who referred to Shahid Madani Hospital in Khorramabad due to the large omphalocele on her chest with pulsating mass above it.

received

April 26, 2018

accepted after revision

December 30, 2018
$10.1055 / \mathrm{s}-0039-1683936$ ISSN 2157-6998.

\section{Case Report}

A 2-hour-old girl, weighing 3,500 g, was referred to Shahid Madani Hospital in Khorramabad due to the large omphalocele on her chest with pulsating mass above it. The baby was the first child of a 24-year-old mother who was born with an uncomplicated vaginal delivery (-Video 1).

\section{Video 1 \\ Video showing case report from different positions. Online content including video sequences viewable at: https://www.thieme-connect.com/products/ ejournals/html/10.1055/s-0039-1683936.}

Her parents were unrelated. Her mothers' pregnancy was uncomplicated and the mother had no health care and medical care during pregnancy. The family history of the disease was negative. The history of abortion, fetal death, and polyhydramnios in the mother was negative. The history of mother's contact with chemicals, toxins, vegetable pests, opium, cocaine, medications (sodium valproate, phenytoin, lithium, lead) radiation, or and environmental factors were negative. In physical examination, the neonatal reflexes were reduced.
Copyright $\odot 2019$ by Thieme Medical Publishers, Inc., 333 Seventh Avenue, New York, NY 10001, USA. Tel: +1(212) 584-4662.

\section{License terms}

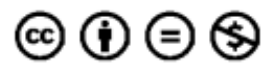


The vital signs of the neonate were: blood pressure 45/30, respiratory rate 72 beat/ min, temperature $36^{\circ} \mathrm{C}$, heart rate $135 / \mathrm{min}$ and $\mathrm{SPO}_{2}=70 \%$, the examination of the head and neck, cyanosis of the lips, and oral mucosa were observed . The baby had tachypnea and grunting. The large omphalocele was seen in the anterior part of chest and abdomen with $20 \times 20 \mathrm{~cm}$ in diameter with a thin membrane. At the top of the omphalocele, a pulsatile mass was seen and in auscultation the heart's grade III/VI murmur was heard. Abdominal viscera were visible inside the omphalocele. The heart was above, the liver in the middle and right, the spleen on the left, the stomach, small intestine, and the colon on the lower part of the masses. The baby was admitted to the intensive care unit. The site of the omphalocele was covered with sterile gas (mesh), and clinical/paraclinical measures were taken. Electrolytes and biochemical test were normal. In the arterial blood gas, there were metabolic acidosis and respiratory acidosis. The thoracoabdominal radiography reported a large omphalocele in the middle line, with the rise of the shadow of the liver and the absence of stomach air and left pulmonary edema. There was no pleural effusion (-Fig. 1).

An echocardiography report ventricular septal defect, aortic arch displacement to right and pulmonary stenosis (Tetralogy of Fallot). Ultrasound reported a large omphalocele in the midline with the movement of the umbilical cord vessels upwards, the left lobe, and parts of the right lobe of the liver, a large part of the spleen on the left, the stomach, parts of the small intestine and colon were in the omphalocele. The gallbladder was not seen. There was no ascitis in the abdomen. Kidneys and bladder were normal. The heart was completely up and out of the chest. The kidneys and bladder were reported with normal size and echo. In the course of admission, due to respiratory failure, the baby needs intuba-

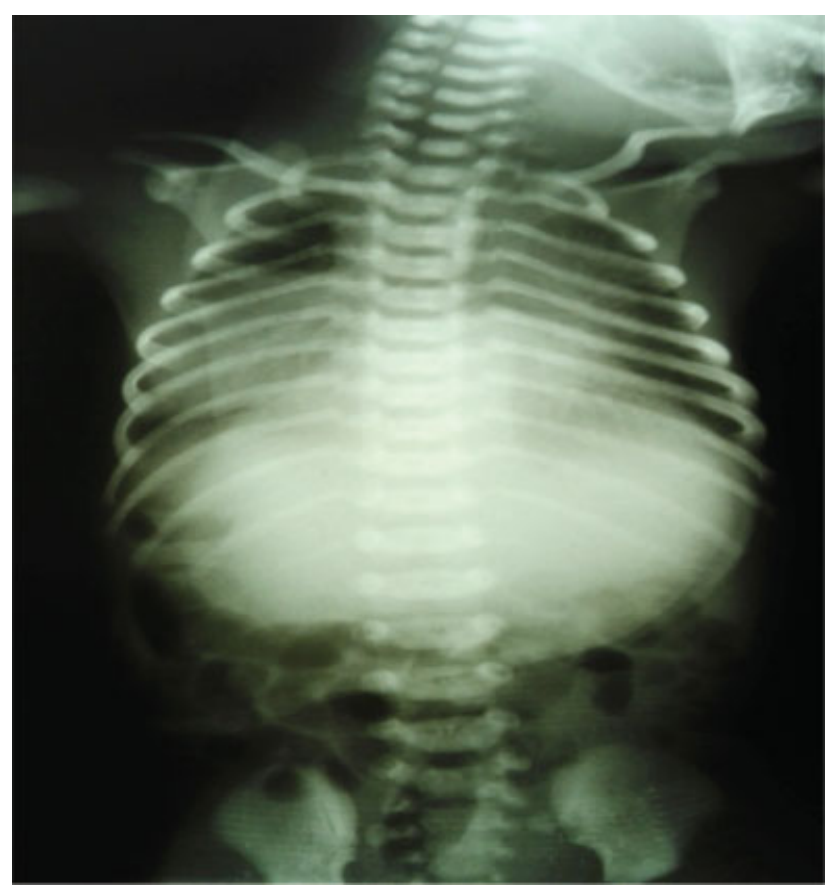

Fig. 1 Simple thoracoabdominal radiography in newborn with PC. PC, Pentalogy of Cantrell.

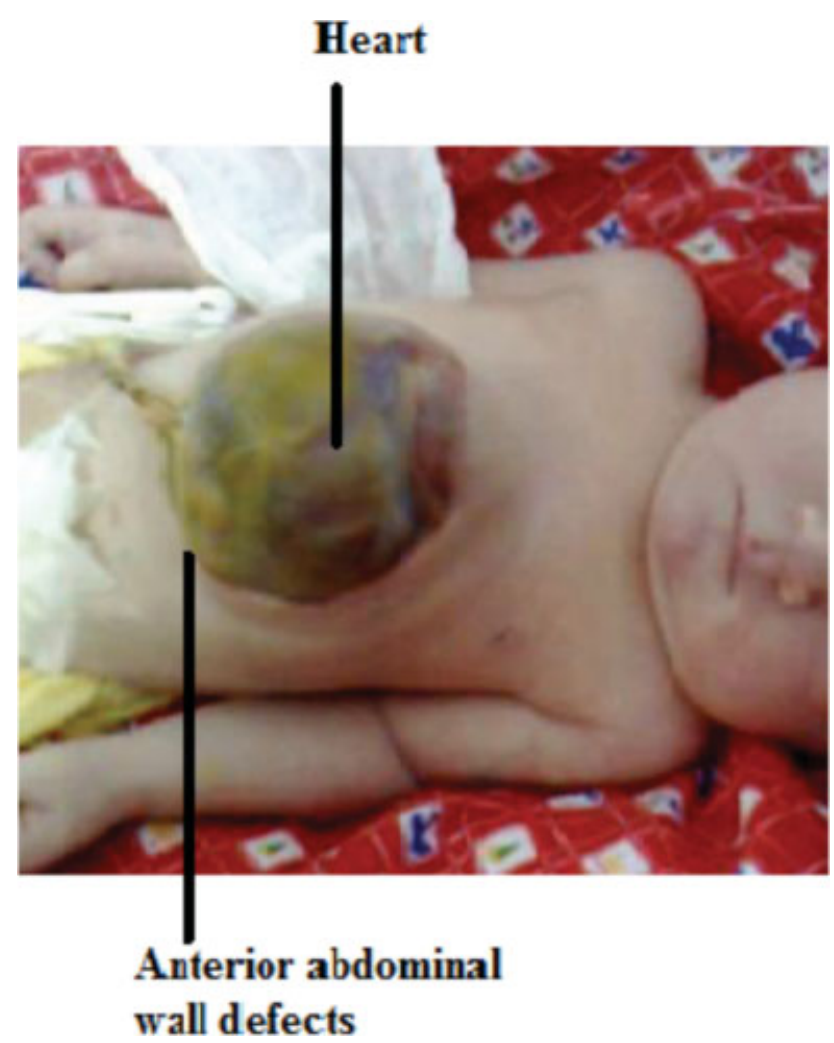

Fig. 2 Anterior view of the infant with PC. PC, Pentalogy of Cantrell.

tion and mechanical ventilation. The baby died after 24 hours because of cardiopulmonary arrest, and unfortunately, the parents did not give autopsy consent (-Fig. 2).

\section{Discussion}

The incidence of PC in most cases was sporadic and reported familial. ${ }^{4}$ Repeat on next pregnancy, associated with trisomy $13,18,21$, and Turner's syndrome were reported. ${ }^{3}$ Heredity attributed to chromosome $\mathrm{X}$ and gene mutation; thoracoabdominal syndrome (TAS) in the Xq25-q26.1 zone has been identified. ${ }^{5}$ The TAS gene plays a role in the sternum fusion, cardiac defects, and the abdominal wall aperture. ${ }^{6}$ New cases of bone morphogenic protein 2 (BMP2) gene mutations have been reported. This gene is responsible for the natural development of midline structures in the fetus. Additionally, mutations in the $A L D H 1 A 2$ gene coded on chromosome 15 are reported to be responsible for the conversion of vitamin A to retinoic acid which plays a very important role in organogenesis during embryonic development.

The frequency of $P C$ is 1 case per 1,000,000 live births and the ratio of male to the female is $2: 1 .^{1,2}$ In 1998 , vazquezJimenez et al, reported 153 cases of PC, 83\% had heart abnormalities, $74.5 \%$ had abdominal wall defects, $60 \%$ had sternum defects, and $57 \%$ had diaphragmatic defects. ${ }^{7}$ The most common cardiac abnormality has been reported as ventricular septal defect.

The accompanying anomalies with PC consist of club foot, cleft lip and palate, spina bifida, meningocele, meningomyelocele, hydrocephalus, and encephalocele. ${ }^{8,9}$ The pathogenesis 
of the disease is unknown; however, the developmental defect of the mesoderm layer on 14 to 18 days leads to the absence of the formation of the horizontal diaphragm wall. This is followed by the migration of visceral mesodermia over the abdomen to the chest, the defective formation of sternum and abdominal wall, and the inclusion of high visceral apertures including the heart, parts of the pericardium which were protrude into the cavity.

Some of the possible causes of pathogenesis include external mechanical transduction to the embryo, external teratogenic agents including the amniotic band resulting in tearing the yolk sac in 14 to 18 days of the onset of conception, alcohol and drug use, and maternal infections during pregnancy. Others include viral infections in the first trimester of pregnancy and the use of chlorine gas in pregnant women. The diagnosis of PC with the help of ultrasound prior to the 12th week of conception is impossible, since at this time, the abdominal viscera are outside the abdominal cavity and in yolk sac. Prognosis of PC is poor and varies depending on the severity of cardiac lesions and associated anomalies. ${ }^{1}$ Deaths following surgery have been reported in cases of cardiac abnormalities of up to $50 \%{ }^{1}$ The main causes of mortalities are hypotension, shock, heart failure, bradycardia, and cardiac arrest.

\section{Conclusion}

The presence of omphalocele in the fetus from 14th week of pregnancy can be detected by ultrasound. Therefore, in all cases with omphalocele, the presence of PC and associated anomalies is recommended.

\section{Compliance with Ethical Standards}

Research involving human participants and/or animalsethical approval: All procedures performed in studies involving human participants were in accordance with the ethical standards of the institutional and/or national research committee and with the 1964 Helsinki declaration and its later amendments or comparable ethical standards.

\section{Informed Consent}

Informed consent was obtained from all individual participants included in the study.

\section{Conflict of Interest}

The authors deny any conflicts of interest in any terms or by any means during the study. All the funds are provided by research center fund and deployed accordingly.

\section{References}

1 Carmi R, Boughman JA. Pentalogy of Cantrell and associated midline anomalies: a possible ventral midline developmental field. Am J Med Genet 1992;42(01):90-95

2 Martin RA, Cunniff C, Erickson L, Jones KL. Pentalogy of Cantrell and ectopia cordis, a familial developmental field complex. Am J Med Genet 1992;42(06):839-841

3 Nayak S, Dash SP, Khatua M. Fetal anomaly: Pentalogy of Cantrell. IOSR J Dent. Med Sci 2015;14(06):52-55

4 Puvabanditsin S, Di Stefano V, Garrow E, Wong R, Eng J, Balbin J. Ectopia cordis. Hong Kong Med J 2013;19(05):447-450

5 Chandran S, Ari D. Pentalogy of cantrell: an extremely rare congenital anomaly. J Clin Neonatol 2013;2(02):95-97

6 Parvari R, Weinstein Y, Ehrlich S, Steinitz M, Carmi R. Linkage localization of the thoraco-abdominal syndrome (TAS) gene to Xq25-26. Am J Med Genet 1994;49(04):431-434

7 Vazquez-Jimenez JF, Muehler EG, Daebritz S, et al. Cantrell's syndrome: a challenge to the surgeon. Ann Thorac Surg 1998; 65(04):1178-1185

8 Jafarian AH, Omidi AA, Fazel A, Sadeghian H, Joushan B. Pentalogy of Cantrell: a case report. J Res Med Sci 2011;16(01):105-109

9 Taee N, Tarhani F, Goodarzi MF, Safdari M, Bajelan A. Mermaid syndrome: a case report of a rare congenital anomaly in full-term neonate with thumb deformity. AJP Rep 2018;8(04):e328-e331 